\title{
Protéases lysosomiales et invasion tumorale
}

Dans de nombreux types de cancers, les cathepsines, qui sont des protéases lysosomiales, apparaissent synthétisées en quantité augmentée et sécrétées de manière exagérée. Dans le cancer du sein, l'hyperproduction de cathepsine $D$ semble être un facteur important de mauvais pronostic, laissant craindre l'apparition de métastases. Les mécanismes de l'hyperexpression et de l'hypersécrétion de la cathepsine $D$ semblent associer une stimulation de la transcription du gène par les œstrogènes et des facteurs de croissance, et une diminution de la concentration de récepteurs du mannose-6-phosphate, entraînant un "déroutage" accru de l'enzyme produite en excès et sa sécrétion dans le milieu extracellulaire.

\section{Henri Rochefort Françoise Capony}

\section{ADRESSE}

H. Rochefort : professeur de biologie cellulaire. F. Capony : chargée de recherche à l'Inserm. Inserm U. 148, hormones et cancer et laboratoire de biologie cellulaire, facultć de médecine, 60, rue de Navacelles, 34090 e rôle majeur des cathepsines est de dégrader les protéines dans les lysosomes [1]. Elles ont également été impliquées dans la maturation physiologique de certains peptides. Récemment, plusieurs études indépendantes suggèrent qu'elles pourraient aussi jouer un rôle dans la dissémination métastatique de certaines tumeurs solides. La liste des protéases proposées pour favoriser les métastases est importante et contraste avec la rareté des faits biologiques ou cliniques indiquant que certaines de ces protéases sont effectivement responsables. De plus, dans la mesure où la plupart des protéases sont sécrétées sous forme de pro-enzymes inactives sur lesquelles agissent parfois des inhibiteurs endogènes, il devient nécessaire d'identifier la (ou les) protéase(s) qui déclenche(nt) la cascade d'activation protéolytique et qui pourraient être réellement asso- ciées, voire responsables, de l'invasion des tissus adjacents par les cellules tumorales. Ce sont surtout les protéases neutres - telles que les activateurs du plasminogène [2] et les collagénases de type IV [3] - qui ont, jusqu'ici, été les plus évoquées comme intervenant dans le déclenchement des processus d'invasion métastatique.

Les études fondamentales faites à partir de lignées de culture de cellules cancéreuses ont récemment introduit la notion qu'il fallait également tenir compte de l'hyperexpression et du déroutage des pro-cathepsines dans le processus d'invasion tumorale $[4,5]$.

Trois cathepsines ont été décrites comme étant hyperexprimées et hypersécrétées par les cellules cancéreuses. Deux protéases à cystéine la pro-cathepsine B dans les cancers de l'ovaire et les mélanomes [4], la pro-cathepsine $\mathrm{L}$ dans les sarcomes et 
les fibroblastes NIH 3T3 transformés par le virus $\mathrm{Ki}$ Ras [6] - et une aspartyl protéase - la cathepsine $\mathrm{D}$, particulièrement étudiée dans les cancers du sein humain $[5,7]$. Nous nous limiterons à cette dernière pour laquelle il existe plusieurs études cliniques récentes indiquant une forte corrélation entre l'incidence des métastases et la concentration de cathepsine D dans les extraits cellulaires de la tumeur primitive.

\section{La maturation normale des cathepsines et leur routage vers les lysosomes}

Les cathepsines sont ubiquitaires, mais leur niveau d'expression peut varier d'une espèce à l'autre, d'un organe à l'autre et même d'un type cellulaire à un autre, suggérant qu'il existc des fonctions plus spécialisées et des mécanismes spécifiques de l'expression de leur gène. Les différentes étapes de la biogenèse du tri et du transport des enzymes lysosomiales sont connues (pour revues : $[8,9])$ et schématisées dans la figure 1. La maturation des pro-cathepsines comprend plusieurs étapes conduisant à la synthèse d'enzymes actives et localisées dans les lysosomes [10]. La première étape est le clivage du peptide signal. La pro-enzyme est ensuite glycosylée ( $\mathrm{N}$-glycosylation) et reconnue par deux enzymes qui assurent la formation du signal mannose-6-phosphate. Dans le réseau trans golgien, le complexe interagit avec les récepteurs mannose-6-phosphate et est transporté dans les endosomes (figure 1) [8,9]. Au niveau des endosomes, l'acidification permet la dissociation de la pro-enzyme lysosomiale qui se dirige vers les lysosomes et du récepteur mannose-6-phosphate qui est recyclé vers le Golgi. Une altération de ce phénomène de

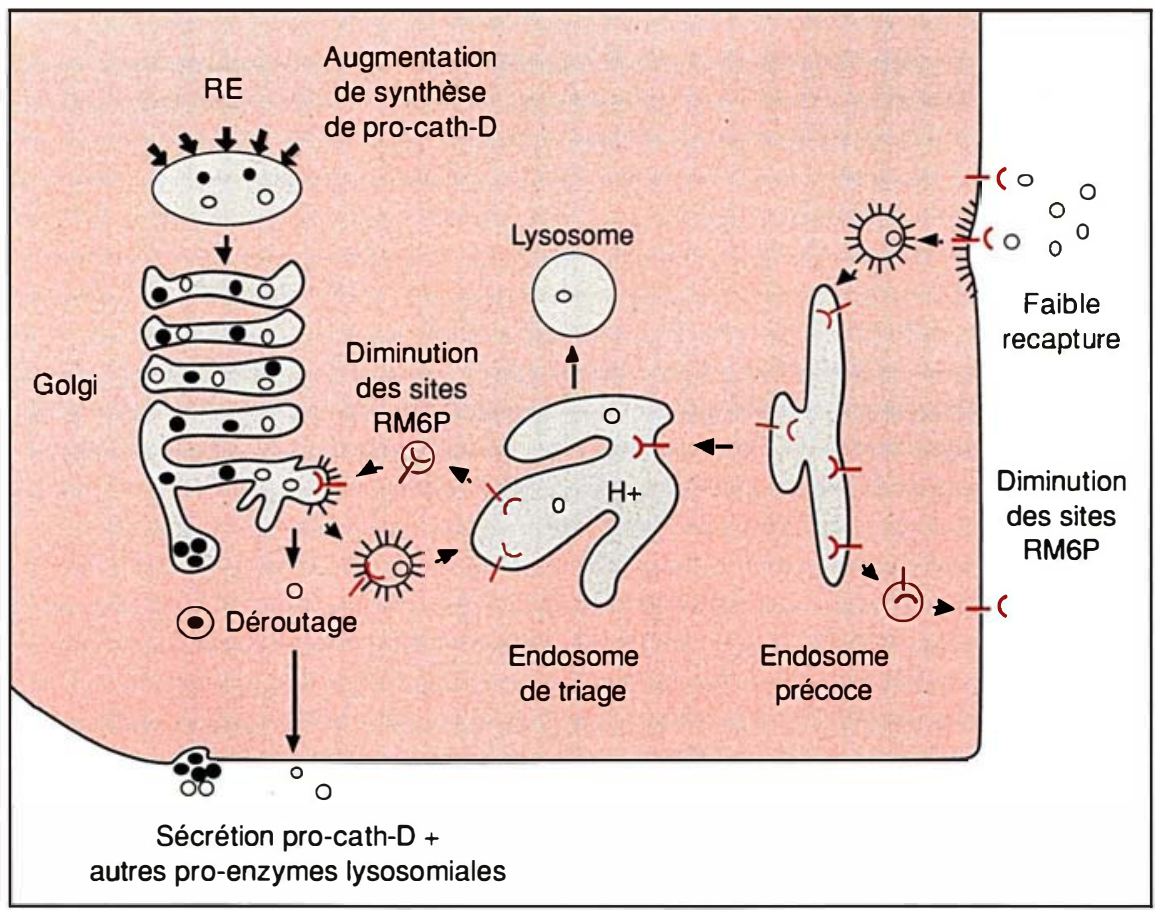

Figure 1. Routage de la cathepsine $D$ via les récepteurs mannose-6-phosphate et déroutage dans les cellules cancéreuses. Le déroutage des enzymes lysosomiales dans les cancers du sein serait dû à la saturation des récepteurs mannose-6-phosphate provoquée par, d'une part, l'hyperexpression du gène cathepsine $D$ entraînant une production accrue de la pro-cathepsine $D$ et, $d$ 'autre part, une diminution de concentration des récepteurs mannose-6-phosphate [14]. Enzymes lysosomiales (o), protéines destinées à la sécrétion $(\bullet)$, récepteur mannose-6-phosphate $R-M 6 P(-C)$. (Figure modifiée d'après Dahms et al. [8], avec la permission de l'éditeur.) routage observée dans certaines maladies héréditaires ( $I$ cell disease, etc.) conduit à une hypersécrétion de certaines pro-enzymes. Parallèlement, ces pro-enzymes subissent plusieurs modifications protéolytiques représentées dans la figure 2, p. 33. Le clivage du pro-fragment dans un compartiment acide [10] est suffisant pour activer l'enzyme.

\section{La cathepsine $D$, une protéase produite en excès et égarée à l'extérieur des cellules cancéreuses}

C'est en étudiant les protéines sécrétées par des lignées cellulaires humaines hormonodépendantes de cancer du sein que notre laboratoire a découvert une glycoprotéine dont la sécrétion était particulièrement stimulée par les œstrogènes (inhibée par les anti-œstrogènes) et produites en excès, de façon constitutive, par les cancers hormono-indépendants [5, 7]. Le développement d'anticorps monoclonaux nous a permis de purifier cette protéine. L'étape décisive dans son identification a été la découverte, sur sa partie glycannique, du signal mannose-6-phosphate spécifique des enzymes lysosomales [11]. Par la suite, la séquence nucléotidique de l'ADNc cloné s'est avérée identique à celle de la pro-cathepsine $\mathrm{D}$ de rein humain, à l'exception d'une mutation sur une des bases de cet ADNc, localisée dans le pro-fragment et correspondant au remplacement Ala par Val (38 acide aminé de la procathepsine D) [12]. Cette mutation est également présente sur le gène correspondant des cellules MCF7 [P. Augereau, non publié] mais n'a pas été retrouvée dans une autre lignée (ZR75-1) [Westley], suggérant qu'elle pourrait correspondre à un polymorphisme. Une étude plus complète sur plusicurs tumeurs mammaires, après amplification du gène par PCR, permettra d'évaluer la fréquence de cette mutation.

Pour déterminer si la sécrétion de la pro-cathepsine D caractérisait les cellulaires mammaires en général ou était spécifique des cellules transformées, nous avons comparé sa production, sa sécrétion et sa structure 


\section{RÉFÉRENCES}

1. Kirschke H, Barrett AJ. Chemistry of lysosomal proteases. In : Glaumann $\mathrm{H}$, Ballard FJ, eds. Lysosomes : their role in protein breakdown. London : Academic Press, 1987 : 193-238.

2. Dano K, Andreasen PA, GrondahlHansen J, Kristensen P, Nielson LS, Skriver L. Plasminogen activators, tissue degradation and cancer. Adv Cancer Res 1985 ; 44: 139-266.

3. Liotta LA, Tryggvason K, Garbisa S Hart I, Foltz CM, Shafie S. Metastatic potential correlate with enzymatic degradation of basement membrane collagen. Nature 1980 ; 284 : 67-8

4. Sloane BF, Dunn JR, Honn KV. Lysosomal cathepsin B : correlation with metastatic potential. Science $1981 ; 212$ : 1151-3. 5. Rochefort H, Capony F, Garcia M, et al. Estrogen-induced lysosomal proteases secreted by breast cancer cells. A role in carcinogenesis ? J Cell Biochem 1987; 35 17-29.

6. Troen BR, Gal S, Gottesman MM Sequence and expression of the cDNA for MEP (major excreted protein), a transformation-regulated secreted cathepsin. Biochem J 1987 ; 246 : 731-5.

7. Westley B, Rochefort H. A secreted glycoprotein induced by estrogen in human breast cancer cell lines. Cell $1980 ; 20$ : 352-62.

8. Dahms NM, Lobel P, Kornfeld S. Mannose-6-phosphate receptors and lysosomal enzymes targeting. J Biol Chem 1989 ; 264: 12115-8.

9. Von Figura K, Hasilik A. Lysosomal enzymes and their receptors. Ann Rev Biochem $1986 ; 55: 167-93$

10. Erikson AH. Biosynthesis of lysosomal endopeptidases. J Cell Biochem 1989; 40 : 31-41.

11. Capony F, Morisset M, Barrett AJ, et al. Phosphorylation, glycosylation and proteolytic activity of the $52 \mathrm{~K}$ estrogen-induced protein secreted by MCF7 cells. J Cell Biol $1987 ; 104: 253-62$.

12. Augereau P, Garcia M, Mattei MG, $e$ al. Cloning and sequencing of the $52 \mathrm{~K}$ cathepsin D cDNA of MCF7 breast cancer cells and mapping on chromosome 11. Mol Endo 1988 ; 2 : 186-92.

13. Capony F, Rougeot C, Montcourrier P, Cavaillès V, Salazar G, Rochefort $\mathrm{H}$. Increased secretion, altered processing, and glycosylation of pro-cathepsin D in human mammary cancer cells. Cancer Res 1989 ; 49: 3904-9.

14. Capony F, Rougeot C, Cavaillès V, Rochefort H. Estradiol increases the secretio by MCF7 cells of several lysosomal proenzymes. Biochem Biophys Res Commun 1990 ; 171: $972-8$ dans des lignées de cellules cancéreuses et dans des cellules épithéliales mammaires normales obtenues après digestion enzymatique des glandes mammaires issues de réductions mammoplastiques [13]. L'ensemble des différences retrouvées entre la cathepsine $\mathrm{D}$ des cellules de cancer du sein et des cellules normales est résumé dans le Tableau I. La concentration cytosolique totale de cathepsine $\mathrm{D}$ et de son ARN messager ainsi que la quantité de précurseur sécrété sont respectivement 8 et 30 fois plus élevées dans des cellules cancéreuses que dans les cellules normales.

Le mécanisme de l'hypersécrétion des cathepsines dans les cellules cancéreuses semble complexe et être différent selon le type de cathepsine (Tableau I). Pour la cathepsine D, un des facteurs majeurs semble être l'hyperexpression du gène qui entraîne la saturation du récepteur mannose-6-phosphate, l'excès de procathepsine $\mathrm{D}$ produite étant alors sécrétée. Nous avons d'ailleurs montré récemment que, dans les cellules MCF7, l'œstradiol entraîne aussi la sécrétion d'autres enzymes lysosomiales sans modifier leur niveau d'ARN messager, et que ce sont les lignées cellulaires cancéreuses contenant le moins de récepteurs mannose-6-phosphate qui sécrètent le plus de procathepsine D [14] (figure 1).

\section{La régulation de l'expression génique de la cathepsine D}

La régulation de l'expression de la cathepsine $\mathrm{D}$ est complexe et variable selon les tissus. L'hyperproduction de la cathepsine $\mathrm{D}$ est secondaire à une accumulation de son ARN messager de 2,2 kb. Le mécanisme
Tableau I

ALTÉRATIONS DES CATHEPSINES DANS LES CELLULES CANCÉREUSES

\begin{tabular}{|c|c|c|c|}
\hline & Cath-D & Cath-B & Cath-L \\
\hline 1. Expression & & & \\
\hline 2. Déroutage & Sécrétée & $\begin{array}{c}\text { Sécrétée } \\
\text { Membranaire? }\end{array}$ & Sécrétée \\
\hline $\begin{array}{l}\text { 3. Mécanisme } \\
\text { du déroutage }\end{array}$ & $\begin{array}{c}\text { Concentration } \\
\text { R-M6P }\end{array}$ & $?$ & $\begin{array}{l}\text { Affinité pour } \\
\text { le R-M6P }\end{array}$ \\
\hline 4. Régulation & $\begin{array}{l}\text { OEstrogènes } \\
\text { Facteurs } \\
\text { de croissance }\end{array}$ & $\begin{array}{c}\text { OEstrogènes } \\
\text { (Utérus normal) }\end{array}$ & $\begin{array}{l}\text { Facteurs de } \\
\text { croissance } \\
\text { Ki-Ras } \\
\text { Phorbol esters }\end{array}$ \\
\hline $\begin{array}{l}\text { 5. Séquence } \\
\text { peptidique }\end{array}$ & $\begin{array}{l}\text { Mutation } \\
\text { Ala } \rightarrow \text { Val } \\
\text { (38॰ acide } \\
\text { aminé) }\end{array}$ & $?$ & $?$ \\
\hline 6. Glycosylation & + acide & $\begin{array}{c}+ \text { glycosylée } \\
+ \text { stable }\end{array}$ & $\begin{array}{c}? \\
+ \text { stable }\end{array}$ \\
\hline $\begin{array}{l}\text { 7. Expression dans } \\
\text { les cancers } \\
\text { cliniques }\end{array}$ & $\begin{array}{l}\text { Cancer du sein } \\
\text { Mélanomes } \\
\text { de l'endomètre }\end{array}$ & $\begin{array}{c}\text { Colon } \\
\text { Ovaire-mélanome }\end{array}$ & $\begin{array}{c}\text { Colon } \\
\text { Pancréas? } \\
\text { Sarcomes }\end{array}$ \\
\hline $\begin{array}{l}\text { 8. Valeur } \\
\text { pronostique }\end{array}$ & $\begin{array}{l}\text { Métastases } \\
\text { Cancers du sein }\end{array}$ & Études en cours & Études en cours \\
\hline
\end{tabular}

Le point commun des cathepsines $D, B$ et $L$ dans les cellules cancéreuses est l'hyperexpression de leur gène, le déroutage et l'hypersécrétion de leur précurseur, et le fait qu'elles soient induites par divers types de mitogène. La nature des hormones qui contrôlent le gène et le mécanisme du déroutage varient selon la cathepsine et le type cellulaire étudiés. Les types de cancer dans lesquels ces modifications ont été décrites varient également selon les cathepsines. R-M6P: récepteur du mannose-6-phosphate. 
de l'induction semble être direct et essentiellement transcriptionnel [12], comme cela a été montré par le laboratoire de $P$. Chambon pour le gène $p S 2$ et le récepteur de la progestérone. Les éléments de réponse aux œstrogènes sont en cours de caractérisation dans les régions 5' du gène cloné de la cathepsine D humaine [P. Augereau et al., en préparation]. L'expression du gène de la cathepsine $\mathrm{D}$ dans les cancers du sein est stimulée non seulement par les œstrogènes, mais aussi indirectement par les facteurs de croissance tels que l'EGF, l'IGF1 et l'insuline [15]. Dans les cellules normales, la régulation du gène de la cathepsine $\mathrm{D}$ est pratiquement inconnue, sauf dans l'endomètre, où c'est la progestérone
- et non plus les œstrogènes - qui est l'inducteur [16]. Cela est particulièrement intéressant car la progestérone induit également la cathepsine L dans l'endomètre de la chatte où cette protéase est supposée pour stimuler la nidation du blastocyte, un autre processus invasif [17].

\section{Intérêt pronostique de la cathepsine D dans les cancers du sein}

Le développement (avec la Sanofi) d'anticorps monoclonaux dirigés contre la pro-cathepsine $\mathrm{D}$ des cellules MCF7, nous a permis de réaliser un dosage immunométrique de la totalité de l'enzyme (précurseur et formes matures) dans divers liquides bio- logiques. La cathepsine D est sécrétée en quantité variable dans les liquides de kystes mammaires et dans les épanchements métastatiques pleuraux, mais elle ne semble pas être un marqueur circulant utilisable en cancérologie mammaire.

En revanche, le dosage de la cathepsine $\mathrm{D}$ totale dans les cytosols de cancer du sein semble avoir une valeur pronostique majeure [18-20]. La cathepsine D n'est pas un marqueur d'hormonodépendance, car il n'y a pas de corrélation entre sa concentration tissulaire et celle des récepteurs des œstrogènes ou de la progestérone. Elle est de plus produite en excès sans être contrôlée par les œstrogènes dans les cancers hormono-indépendants. Il existe à ce

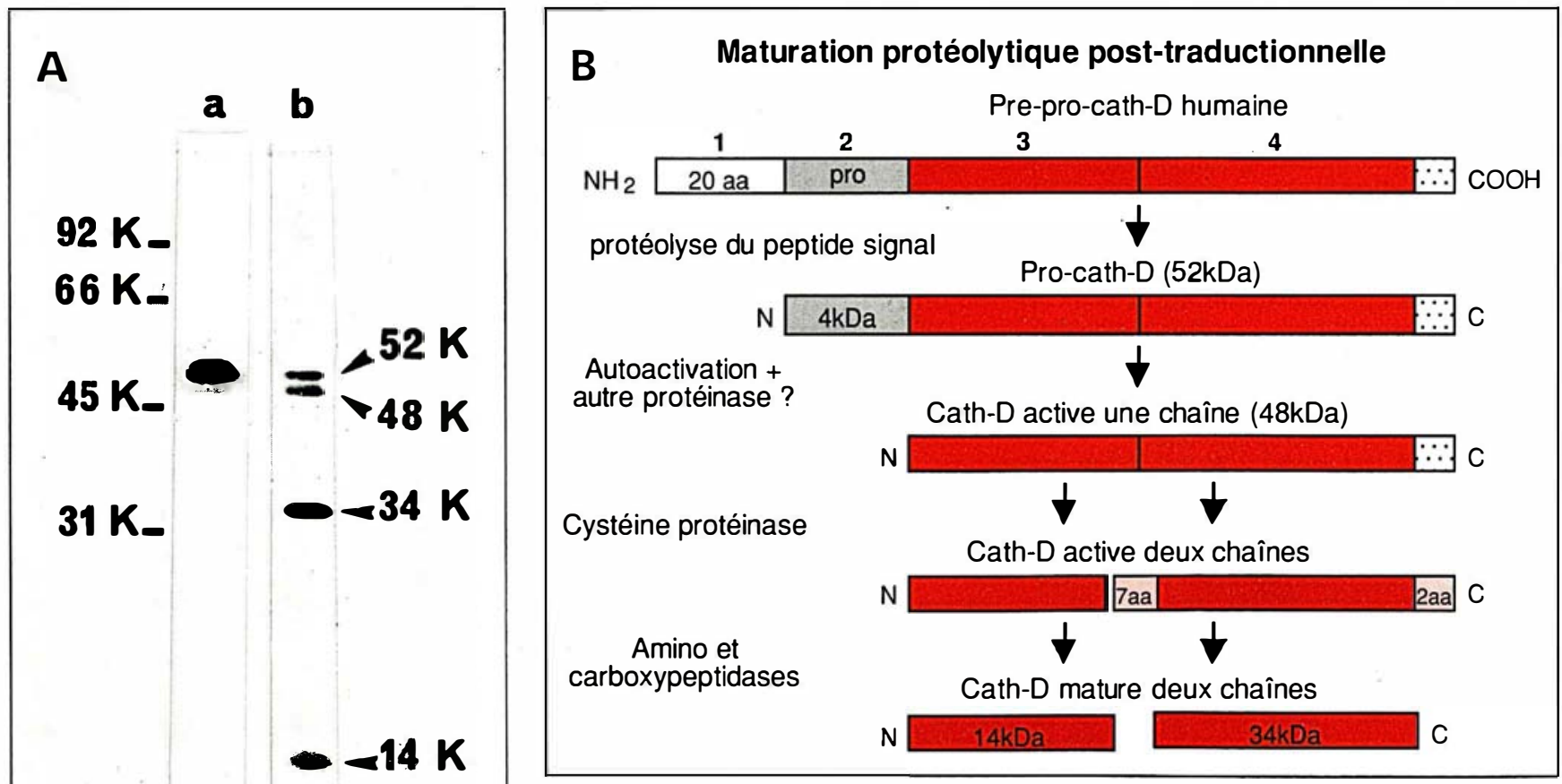

Figure 2. Maturation de la pro-cathepsine $D$ en ses diverses formes actives. A : électrophorèse en gel de polyacrylamide de la cathepsine $D$ purifiée à partir de cellules de cancer du sein MCF7. a) forme sécrétée $52 \mathrm{kDa}$; b) formes cellulaires. Les protéines sont révélées par coloration à l'argent. B : schéma de la maturation de la cathepsine D. L'activité est assurée par la protéolyse du pro-fragment et le site actif est formé par la proximité des deux acides aspartiques, assuré soit par le repliement des formes intermédiaires 51 ou 48 $k D a$, soit par l'association des deux chaînes de la forme mature. Une carboxypeptidase et une aminopeptidase complètent la maturation des deux chaînes. La nature des protéases participant à ces diverses étapes de maturation est indiquée à gauche. 


\section{RÉFÉRENCES}

15. Cavaillès V, Garcia M, Rochefort $H$ Regulation of cathepsin D and pS2 gene expression by growth factors in MCF7 human breast cancer cells. Mol Endocrinol $1989 ; 3: 552-8$

16. Elangovan S, Moulton BC. Progesterone and estrogen control of rates of synthesis of uterine cathepsin D. I Biol Chem $1980 ; 255: 7474-9$

17. Jaffe RC, Donnelly KM, Mavrogianis PA, Verhage G. Molecular cloning and characterization of a progesterone-dependent cat endometrial secretory protein complementary deoxyribonucleic acid. Mol Endocrinol $1989 ; 3$ : 1807-14.

18. Rochefort H. Cathepsin D in breast cancer. Breast Cancer Res Treat 1990 ; 16 $1-13$

19. Spyratos F, Maudelonde $\mathrm{T}$, Brouillet JP, et al. Cathepsin D : an independent prognostic factor for metastasis of breast cancer. Lancet 1989; 8672 : 1115-8. 20. Tandon AK, Clark GM, Cham ness GC Chirgwin JM, McGuire WL. Cathepsin D and prognosis in breast cancer. N Engl J Med 1990; 322 : 297-302. 21. Garcia M, Salazar-Retana G, Pagc̀s A et al. Distribution of the $\mathrm{Mr} 52,000$ estrogen-regulated protein in benign breast diseases and other tissues by immunohistochemistry. Cancer Res 1986 ; 46 : 3734-8. 22. Briozzo P, Morisset M, Capony F, Rougeot $\mathrm{C}$, Rochefort $\mathrm{H}$. In vitro degradation of extracellular matrix with $\mathrm{Mr} 52,000$ cathepsin D secreted by breast cancer cells. Cancer Res 1988 ; 48 : 3688-92.

23. McCoy K, Gal S, Schwartz RH, Gottesman MM. An acidic protease secreted by transformed cells interferes with antigen processing. J Cell Biol 1988; 106 : 1879-84

24. Diment S, Martin KJ, Stahl PD. Cleavage of parathyroid hormone in macrophage endosomes illustrates a novel pathway for intracellular processing of proteins. $J$ Biol Chem 1989 ; 264 : 13403-6.

25. Montcourrier P, Mangeat P, Salazar G Morisset M, Sahuquet A, Rochefort $\mathrm{H}$. Cathepsin D in breast cancer cells can digest extracellular matrix in large acidic vesicles. Cancer Res 1990 ; 50 : 6045-54

26. Morgan DO, Edman JC, Strad ring DN, et al. Insulin-like growth factor II receptor is a multifunctional binding protein. Nature 1987 ; 329 : 301-7.

27. Vignon F, Capony F, Chambon M, Freiss G, Garcia M, Rochefort H. Autocrine growth stimulation of the MCF7 breast cancer cells by the estrogen-regulated $52 \mathrm{~K}$ protein. Endocrinology $1986 ; 118$ : $1537-45$.

28. Pietras RJ, Szego CM. Surface modifications evoked by estradiol and diethylstilbestrol in isolated endometrial cells : evidence for lectin probes and extracellular release of lysosomal protease. Endocrinology

\begin{tabular}{|c|c|c|c|}
\hline $\begin{array}{r}\text { ENSEMB } \\
\text { INDIQUAN } \\
\text { DU DOSAGE DE LA CA }\end{array}$ & $\begin{array}{l}\text { Tableau } \\
\text { LLE DES ÉTU } \\
\text { T UNE VALE } \\
\text { THEPSINE D }\end{array}$ & $\begin{array}{l}\text { DES CLIN } \\
\text { UR PRON } \\
\text { DANS LE }\end{array}$ & $\begin{array}{l}\text { JQUES } \\
\text { OSTIQUE } \\
\text { ES CANCERS DU SEIN }\end{array}$ \\
\hline Références & Centres & $\begin{array}{l}N^{\circ} \text { des } \\
\text { patients }\end{array}$ & Informations \\
\hline $\begin{array}{l}\text { 1. Maudelonde et al. } \\
\text { Cancer Res } 1988 ; 48 \text { : } \\
462 \text {. }\end{array}$ & Montpellier & 183 & $\begin{array}{l}\text { Un marqueur indépendant } \\
\text { des autres facteurs de } \\
\text { pronostic }\end{array}$ \\
\hline $\begin{array}{l}\text { 2. Thorpe et al. } \\
\text { Cancer Res } 1989 ; 49 \text { : } \\
6008 .\end{array}$ & Copenhague & 400 & $\begin{array}{l}\text { Diminution de la survie } \\
\text { sans rechute chez les } \\
\text { patientes pré- et post- } \\
\text { ménopausées }\end{array}$ \\
\hline $\begin{array}{l}\text { 3. Spyratos et al. } \\
\text { The Lancet } 1989 \text {; ii : } \\
1115 .\end{array}$ & Saint-Cloud & 120 & $\begin{array}{l}\text { Augmentation des méta- } \\
\text { stases, que les ganglions } \\
\text { axillaires soient envahis ou } \\
\text { non }\end{array}$ \\
\hline $\begin{array}{l}\text { 4. Tandon et al. } \\
N \text { Engl J Med 1990; } \\
\text { 322:297. }\end{array}$ & San Antonio & 200 & $\begin{array}{l}\text { Diminution de la survie en } \\
\text { l'absence de ganglions } \\
\text { envahis (anticorps diffé- } \\
\text { rents) }\end{array}$ \\
\hline $\begin{array}{l}\text { 5. Brouillet et al. } \\
\text { Eur J Cancer } 1990 ; 26 \text { : } \\
\text { 437-41. }\end{array}$ & Montpellier & 140 & $\begin{array}{l}\text { Facteur indépendant de } \\
\text { l'amplification des onco- } \\
\text { gènes erb-B2 et int-2 cor- } \\
\text { rélée avec celle des } c-m y c\end{array}$ \\
\hline $\begin{array}{l}\text { 6. Duffy et al. Clin Chem } \\
1991 \text {; (sous presse). }\end{array}$ & Dublin & 128 & $\begin{array}{l}\text { Diminution de la survie } \\
\text { sans rechute et légère cor- } \\
\text { rélation avec l'urokinase }\end{array}$ \\
\hline $\begin{array}{l}\text { 7. Romain et al. } \\
\text { Bull Cancer } 1990 ; 77 \text { : } \\
\text { 439-47. }\end{array}$ & Marseille & 85 & $\begin{array}{l}\text { Survie diminuée chez les } \\
\text { malades avec ganglions } \\
\text { envahis }\end{array}$ \\
\hline $\begin{array}{l}\text { 8. Namer et al. } \\
\text { (soumis pour publication) }\end{array}$ & Nice & 237 & $\begin{array}{l}\text { Survie diminuée chez les } \\
\text { malades avec ganglions } \\
\text { envahis }\end{array}$ \\
\hline $\begin{array}{l}\text { 9. Henri et al. } \\
\text { Cancer } 1990 ; 65 \text { : } \\
\text { 265-71. }\end{array}$ & Newcastle & 94 & $\begin{array}{l}\text { Par immunohistochimie } \\
\text { avec anticorps différents } \\
\text { valeur de bon pronostic }\end{array}$ \\
\hline
\end{tabular}

Le dosage de la cathepsine $D$ a été obtenu dans les cytosols de cancers du sein sauf pour l'étude 9. Les deux mêmes anticorps monoclonaux ont été utilisés sauf pour les études 4 et 9 lanticorps polyclonaux reconnaissant l'enzyme mature $34 \mathrm{kDa}$ pour l'étude 4 et la cathepsine $D$ totale pour l'étude 9).

jour quatre études rétrospectives différentes indiquant toutes, et de façon indépendante, que de fortes concentrations de cathepsine D sont associées à un risque accru de métastases dans les cancers du scin (Tableau II) (figure 3). La valeur prédictive est également valable pour les tumeurs sans envahissement ganglionnaire, ce qui est particulièrement important, car $30 \%$ de ces tumeurs métastasent. Dans une étude multiparamé- trique selon le modèle de Cox, le centre René-Huguenin (Saint-Cloud) a montré que la cathepsine $\mathrm{D}$ arrivait en première position comme test pronostique, avant même la détermination de l'envahissement ganglionnaire (figure 3) [19]. La séric de 400 malades effectuée à San Antonio [20], pour laquelle la concentration de cathepsine D mature a été évaluée par immunoblot à l'aide d'anticorps polyclonaux, confirme nos études et 
indique que dans les tumeurs sans envahissement ganglionnaire $\left(\mathrm{N}^{-}\right)$la cathepsine $\mathrm{D}$ s'avère être le meilleur facteur de pronostic, y compris pour le taux de survie réelle, avant les facteurs classiques. En pratique, le dosage de la cathepsine $\mathrm{D}$ est réalisable sur une aliquote $(50 \mu l)$ d'un cytosol préparé en routine pour le dosage des récepteurs hormonaux. Le dosage utilisé dans le kit ELSA-cathD, distribué par CIS-International, évalue la concentration totale en cathepsine D. Sur la base des premières études disponibles, un taux inférieur à $30 \mathrm{pmol} / \mathrm{mg}$ de protéines cytosoliques indique un pronostic favorable, alors qu'un taux supérieur à $70 \mathrm{pmol} / \mathrm{mg}$ de protéines indique un pronostic réscrvé. Cependant, le seuil critique varie selon les études et un plus grand nombre d'études cliniques sera nécessaire pour le préciser.

Le dosage de la cathepsine $\mathrm{D}$ dans d'autres cancers et dans certaines lésions précancéreuses pourrait être également utile. La concentration de cathepsine D dans certaines mastopathies canalaires prolifératives ou kystiques à risque semble être très élevée, comme l'indiquait une étude immunohistochimique [21].

\section{Hyperproduction de cathepsine $D$ : cause ou conséquence du processus métastatique?}

La signification biologique de fortes concentrations de cathepsine $\mathrm{D}$ dans les cancers du sein canalaires qui développeront des métastases n'est pas élucidée. Il est possible que cette hyperproduction soit un épiphénomène associé à un autre processus responsable des métastases; par exemple, les facteurs de croissance peuvent, en parallèle, induire cette protéase et stimuler la croissance des cellules métastatiques. Alternativement, cette protéase pourrait être directement impliquée dans le processus invasif. Deux grands types de mécanismes peuvent être proposés, selon qu'ils font intervenir l'activité protéolytique de l'enzyme ou sa capacité d'interaction avec les récepteurs mannose-6-phosphate. Le nombre de substrats potentiels de la $\mathrm{m} / \mathrm{s} n^{\circ} 1$, vol. 7, janvier 91

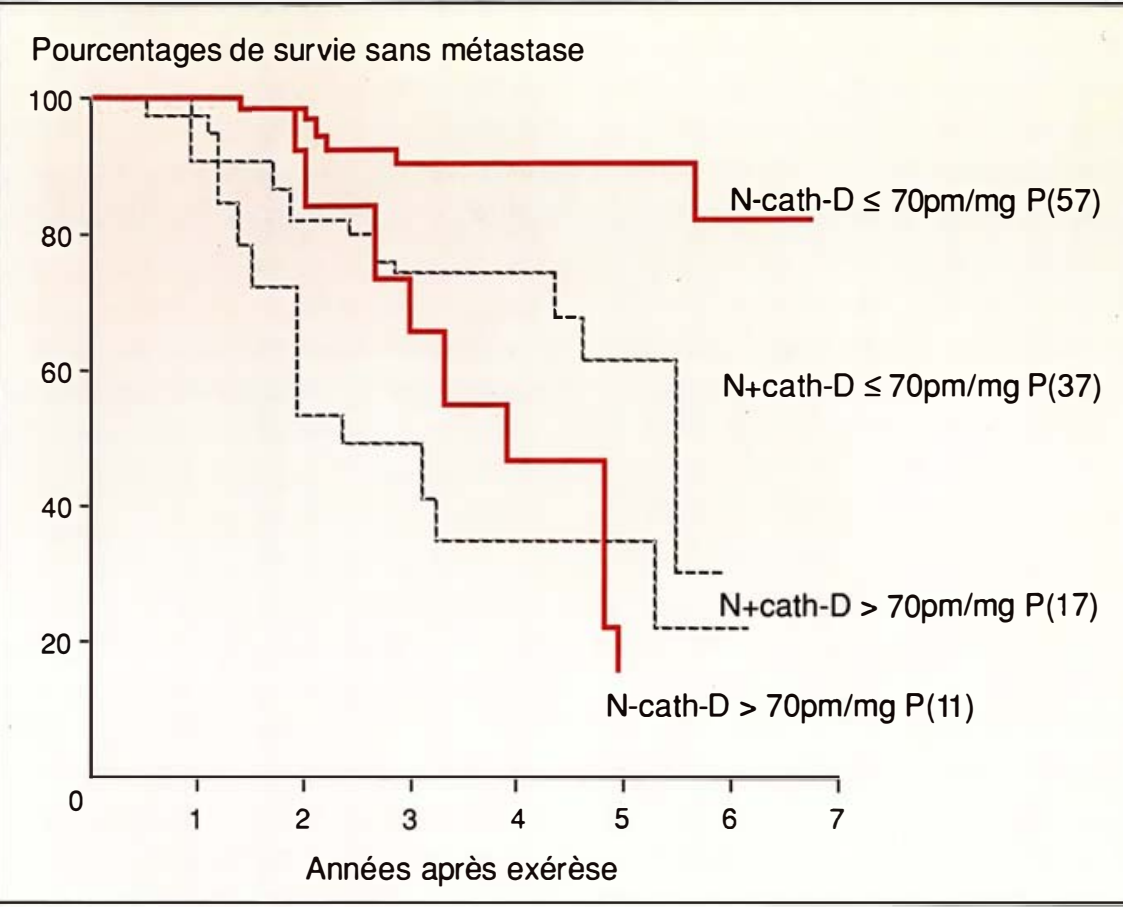

Figure 3. Étude rétrospective de l'intérêt pronostique de la cathepsine D dans les cytosols de cancer du sein. Sur 122 malades, le pourcentage de survie sans métastases est significativement plus faible dans les cancers du sein à forte concentration de cathepsine $D / p<1 \times 10^{-3} d^{\prime}$ après le test de Log-rank). La valeur prédictive est particulièrement significative dans les cancers sans envahissement ganglionnaire $\left(N^{-}\right)$. Le nombre de malades dans chacune des quatre séries est indiqué entre parenthèses. (D'après F. Spyratos et al. [19] avec la permission de l'éditeur.)

cathepsine D est illimité, car cette protéase a une spécificité très large. On sait qu'in vitro, elle est responsable de la dégradation de la matrice extracellulaire [22], qu'elle peut être à l'origine du déclenchement d'une cascade de protéolyse en activant des pro-cathepsines et/ou en dégradant des inhibiteurs de protéases. Elle pourrait activer des précurseurs de facteurs de croissance (TGF $\beta$, TGF $\alpha$, FGFs, etc.) ou des récepteurs de ces facteurs de croissance, modifier des protéines de surface conduisant à une altération des interactions intercellulaires ou, enfin, interférer dans le processus de présentation des antigènes par le complexe majeur d'histocompatibilité à la surface des cellules tumorales [23], etc.

On ignore où la cathepsine $\mathrm{D}$ digérerait ces substrats. Cette protéase ne semble fonctionner que dans un environnement acide $(\mathrm{pH}<5,5)$. Un tel environnement pourrait être localisé à la surface des cellules tumorales au contact de la membrane basale, comme cela existe pour les ostéoclastes et les macrophages adhérant à la matrice extracellulaire, mais cela n'a pas été démontré. Plus évidents sont les compartiments acides intracellulaires, où s'accumulent - dans les cellules cancércuses - la cathepsine D, la matrice extracellulaire endocytosée, et d'autres substrats potentiels et dans lesquels les cathepsines ont été démontrées comme responsables de l'activation de certaines prohormones [24]. De tels compartiments endosomaux acides pourraient être particulièrement augmentés en taille et en nombre dans les cellules des cancers du sein [25].

La deuxième possibilité est que la pro-cathepsine $\mathrm{D}$ intervienne en interagissant et en activant un récepteur membranaire tel que le récepteur du mannose-6-phosphate qui est également le récepteur de l'IGFII 
[26]. Le rôle mitogène de ce récepteur, également localisé à la surface des cellules cancéreuses, n'est pas exclu. L'effet mitogène autocrine de la pro-cathepsine $\mathrm{D}$, observé en culture de cellules MCF7, pourrait donc aussi avoir ce récepteur comme intermédiaire [27].

Des altérations voisines ont également été décrites par d'autres auteurs concernant les cathepsines B et $\mathrm{L}$ (Tableau II). Bien qu'il s'agisse de cancers différents, il existe des points communs troublants tels que l'hyperexpression des gènes, le déroutage et la sécrétion des pro-cathepsines et leur régulation par divers types d'hormones [28] ou facteurs généralement mitogènes. Le rôle éventuel de ces cathepsines dans un processus métastatique a également été invoqué. Si cette hypothèse se confirmait, on pourrait alors envisager de nouvelles voies thérapeutiques visant à traiter ou prévenir les métastases par des antiprotéases spécifiques, des anticorps ou des ARN antisens. Il existe cependant une limitation importante à ce type de traitement, due au fait que les protéines des cellules cancéreuses ont une structure quasi identique à celle des cellules normales. Il semble que dans les cellules de cancers du scin, l'anomalie réside dans l'hyperexpression d'une protéase de structure par ailleurs normale. La cause semble donc être à rechercher au niveau d'une dysrégulation du gène de la cathepsine $D$. Contrairement à l'utilisation clinique de la cathepsine $\mathrm{D}$ comme marqueur pronostique dans les cancers du sein, qui pourrait entrer dans la pratique courante assez rapidement, les applications thérapeutiques visant à bloquer l'action de cette protéase paraissent ainsi encore lointaines

\section{TIRÉS A PART}

H. Rochefort.

\section{Summary}

Lysosomal proteinases and tumor invasion

Alterations of cathepsins B, L and $\mathrm{D}$ in different cancers and virally transformed cells suggest that these lysosomal proteinases might play a role in tumor invasion and metastasis processes. These alterations involve gene overexpression, derouting and increased secretion of these proteases which also are regulated by hormones and mitogenic factors. In this review, we describe more particularly the alteration of cathepsin D in breast cancer cells, and the clinical significance of cathepsin D as a tissue marker for predicting relapse and metastasis. The possible mechanisms by which the overexpression and hypersecretion of cathepsin in cancer cells might facilitate metastasis are discussed. 